\title{
The Effects of UAV Quadcopter Propeller Tilt Angle on Flight Stability
}

\author{
Albert A. Espinoza, MSME, Katherine V. Carrasquillo, José Pérez, Yamil Fernández, Renier Martínez \\ Universidad Ana G. Méndez - Recinto de Gurabo, Puerto Rico, espinozaa1@uagm.edu, kcarrasquillo62@email.uagm.edu, \\ jperez88@email.uagm.edu, yfernandez22@email.uagm.edu, rmartinez190@email.uagm.edu
}

\begin{abstract}
Unmanned Aerial vehicles (UAVs) have captivated the interest of many researchers in recent years and are currently used in a wide range of applications such as: geographic mapping, weather forecasting, aerial photography, law enforcement, and search and rescue. Many of these applications require a multirotor $U A V$ in order to maintain a stationary position in mid-air, and thus UAVs must be designed to be as stable as possible to reduce the need for excessive flight controller corrections and positioning errors, particularly under windy conditions. This research focuses on performing an empirical study on the effects of adding an inward propeller tilt angle, a design feature commonly used to improve stability in fixed-wing aircraft, on the flight stability of a quadrotor UAV. A ready-to-fly quadrotor UAV kit was assembled, and its propellers were tilted inward at various fixed angles of $0^{\circ}, 5^{\circ}$ and $10^{\circ}$, and the planar motion of the UAV was computed using object visual tracking software. The results demonstrate that adding a propeller tilt angle improves quadrotor UAV flight stability.
\end{abstract}

\section{INTRODUCTION}

Unmanned Aerial Vehicles (UAVs), particularly the quadcopter-type configurations, are used quite extensively in a wide range of industries: from agriculture, mining, search and rescue, remote structural inspections, among others [1], [2]. Many of these applications rely on having the quadcopter accurately and safely maintain its attitude and position fixed in space to allow accurate positioning of its end-effector (e.g., camera, actuators, gripper, etc.). Aside from using highprecision (and often costly) sensors, the geometry of the quadcopter frame could be modified to improve its flight stability, particularly during station-keeping tasks. One way this may be accomplished is by adding an inward tilt angle to the propelling system of the quadcopter. This design feature, referred to as a dihedral angle, is commonly used in the aerospace industry to improve the roll stability of an airplane during banking [3]. This upward angle in the wings of an airplane creates a change in lift which generates a restoring rolling moment that rotates the plane back to level position and is normally found in commercial aircraft in which minimal aviation manoeuvring is desired. Tilting the propellers of a quadcopter UAV has been worked extensively in the development of Vertical Take-Off and Landing (VTOL) UAVs in [5] and [6], and to achieve improved UAV controllability in [7]. However, in terms of its applicability improve flight stability in UAV quadcopters, there are fewer and conflicting results presented in the research. Efraim, et al. [8] and Hadayatpour [9] have used this technique in previous research and have shown that tilting the propellers inward in a quadcopter design creates a damping effect that can aid in flight stability during autonomous hovering.. However, Bezerra, et al., presented, albeit in simulation, that tilting the propellers inward does not significantly affect the overall position of the quadcopter UAV [9]. Given that, to the author's knowledge, there are no definitive results related to the effects of tilting the propellers of a quadcopter UAV on flight stability, particularly in station-keeping missions, this study focuses on the development of an empirical study to determine if implementing this design feature leads to improved station-keeping using open-source flight navigation controllers typically used in the UAV industry.

The rest of the paper is organized as follows. In Section II, the structure of the methodology used to estimate UAV position and flight stability are formally presented. Section III is dedicated to the description of the hardware and software used in the experimental studies. In Section IV, the results from the experimental studies are presented and discussed. Finally, Section V provides concluding remarks, as well as any possible future work.

\section{METHODOLOGY}

To measure its position during flight, the UAV was flown to a predefined altitude and then commanded to maintain an autonomously set, fixed position while in the field of view of a video camera, see Fig. 1. The video feed from the camera was processed using MATLAB's Image Processing and Computer Vision toolboxes to keep track the UAV's position. The object tracking algorithm used to determine UAV position is presented in Fig. 2. First, the camera video feed was processed frame by frame and converted to a grayscale image to reduce image processing time.
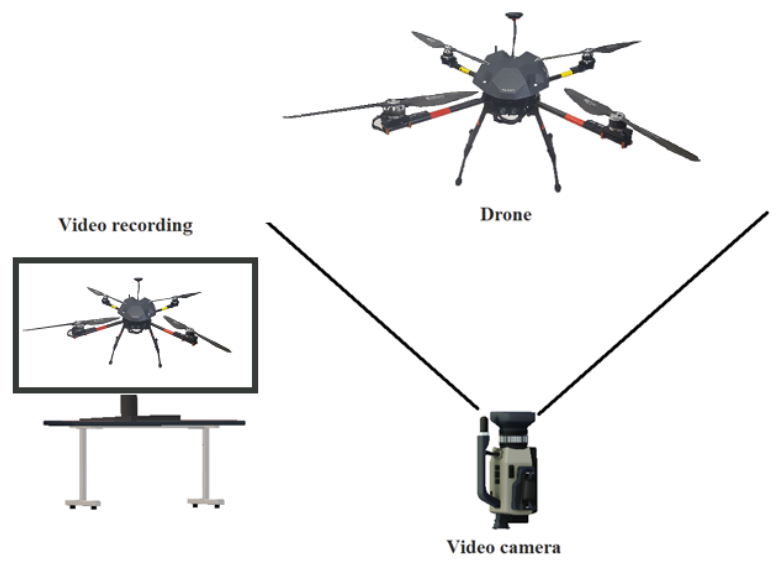

Fig. 1. Urone I rackıng Experımental Setup 


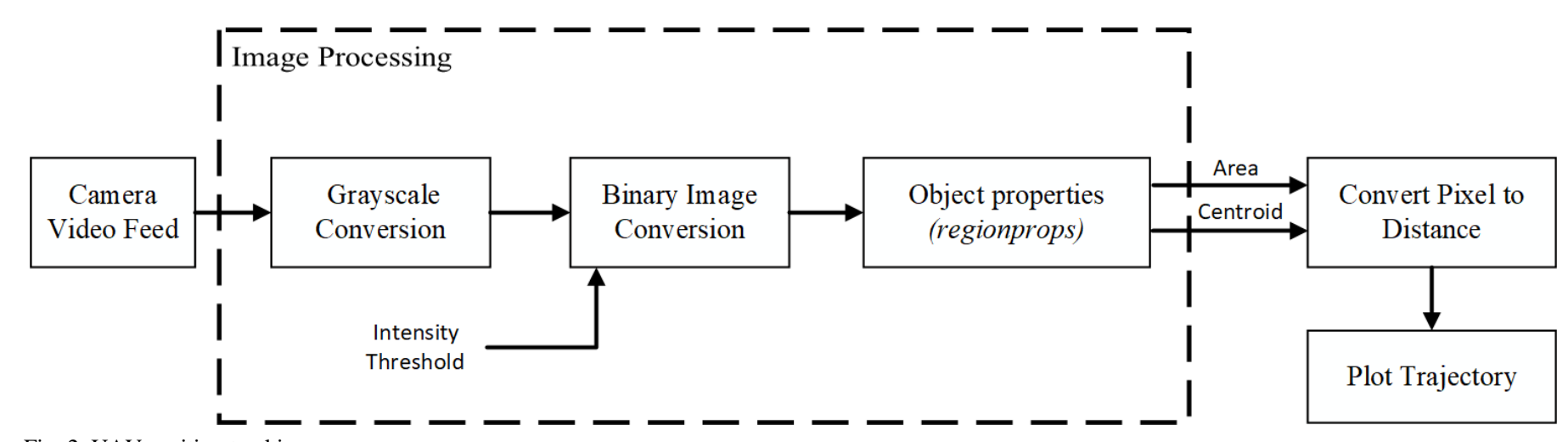

Fig. 2. UAV position tracking process

The grayscale images have an 8-bit intensity value assigned to each pixel, which ranges from 0 to 255 , where 0 defines a black pixel and 255 defines a white pixel. To further simplify object detection, the grayscale image was further converted into a black and white binary image, based on a predefined pixel intensity threshold in the grayscale image, which was varied according to the characteristics of the video, such as the time of day or the relative amount of light entering the camera lens. In the binary image, dark pixels were assigned a value of 0 and lighter pixels were assigned a 1 . This process greatly reduced computation times and improved the object detection process.

After the binary image conversion, the UAV is defined in the image as a collection of interconnected black pixels, which is referred to as a region. Using MATLAB's regionprops function, the total area (defined in squared pixels) occupied by the UAV and the location coordinates (defined in pixels) of the UAV centroid was calculated. Finally, the location of the UAV was converted into real-length units (centimeters) by using the pixel-measurements of known parts of the UAV, such as its arms and landing gear feet, to calculate the scale of the image. This process was repeated frame by frame for the entire duration of the test video in order to generate the plot of the complete trajectory of the UAV.

Furthermore, in order to provide a comparable metric of the relative flight stability of the UAV, the sum of the squared error (SSE) was computed as follows:

$$
\operatorname{SSE}=\sum_{i=1}^{N}\left(D_{i}\right)^{2}
$$

Where $D_{i}$ represents the distance from the location of the UAV centroid at frame $i$ to the UAV centroid location at frame 1 (i.e., the initial position of the UAV). As calculated, this metric provides a measure of the deviation of the UAV's centroid location using the initial position as the desired location. Higher SSE values can be roughly interpreted as a higher variation in centroid location, and thus a relatively less stable station-keeping UAV.

\section{EXPERIMENTAL SETUP}

The experiments were carried out using an off-the-shelf quadcopter UAV frame kit outfitted with a Pixhawk 4 flight controller unit., see Fig. 3 . The Pixhawk 4 processes the data from various navigation sensors, including a high-resolution barometer, two independent triaxial accelerometers, magnetometers, and gyroscopes, as well as GPS/GNSS position data and fuses all sensor data using an Extended Kalman Filter (EKF) to provide accurate UAV positioning and attitude data suitable for autonomous navigation.. In addition to processing all navigation sensor data, the NCU also processes PPM/SBUS radio input signals commonly used in radio control applications and can send out up to 8 independent PWM signals to control external motors, servos, or other peripherals. All RC I/O data is processed by a dedicated processor.

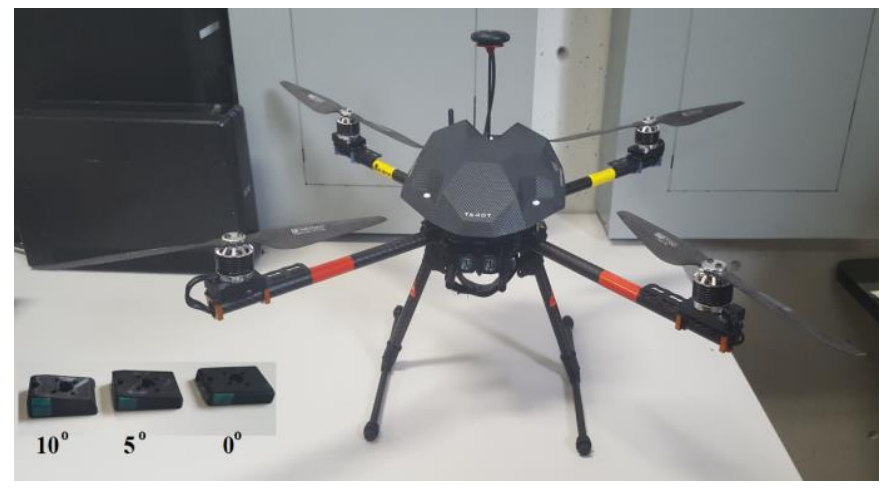

Fig. 3. Quadcopter UAV Kit Drone and propeller tilt adapters

The Pixhawk 4 is also configured to run ArduPilot, an open source, full-featured autopilot software commonly used in programming autonomous UAV systems. ArduPilot is used to process the control input provided by the UAV operator to the motors and logs all sensor data from the Pixhawk 4. Fig. 4 presents a block diagram of the internal UAV hardware components used in the experiments. 


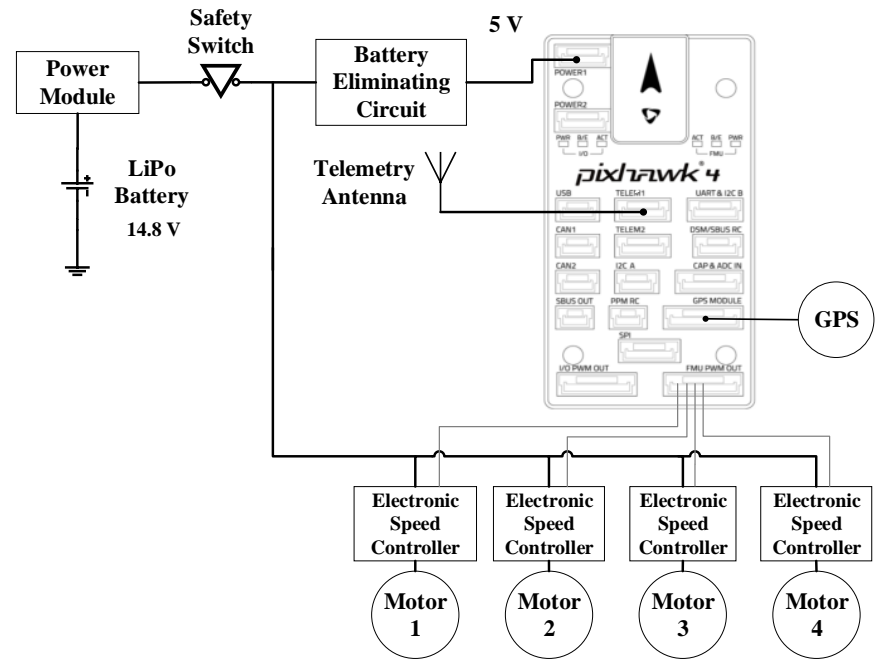

Fig. 4. UAV Hardware Components (Block Diagram)

Mission Planner was used as the ground station application to communicate with the ArduPilot software, configure all the navigation-relevant parameters, and calibrate the on-board sensors. See Fig. 5. Additionally, Mission Planner also provides runtime telemetry from all onboard sensors and communicates with the ArduPilot software using Micro Air Vehicle Link (MAVLink) communication protocol. The video camera used was a 60 frame per second, $1980 \mathrm{x}$ 1080 pixel resolution GoPro® ${ }^{\circledR}$ camera.

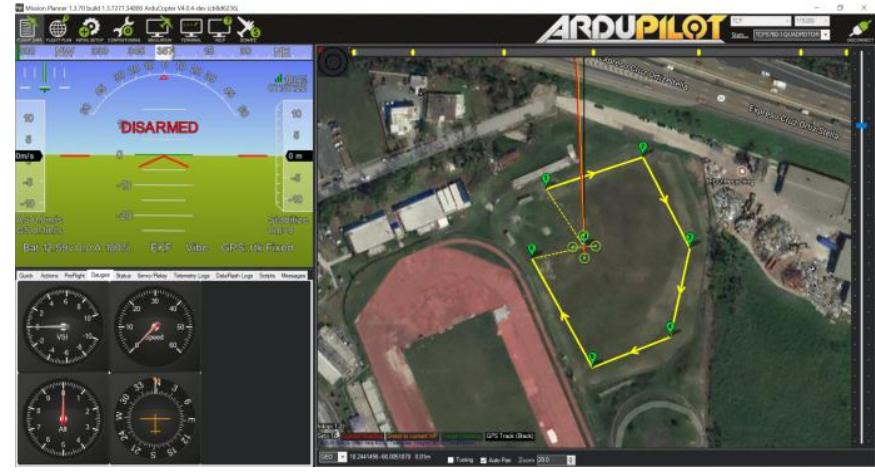

Fig. 5. Mission Planner Ground Station Application

\section{ANALYSIS OF RESULTS}

All tests were performed outdoors by flying the drone in station-keeping (i.e., loiter) mode for all three different propeller tilt angles of $0^{\circ}, 5^{\circ}$, and $10^{\circ}$. For each test, a 30 second sample video was taken and processed as discussed in Section II to plot the overall station-keeping trajectory of the UAV. To ensure that the object tracking algorithm was working properly, the UAV centroid location was overlayed over the video. A sample frame of the overlay is shown in Fig. 6. For reference, the initial and final positions of the UAV centroid are also indicated.
The UAV trajectories for each tilt angle are presented in Fig. 7. The data demonstrates that the UAV centroid drifted away from its initial position significantly, but generally stayed within a window of $60 \mathrm{~cm}$ by $90 \mathrm{~cm}$. In all three cases the UAV tended to sway towards the right side, potentially due to wind.

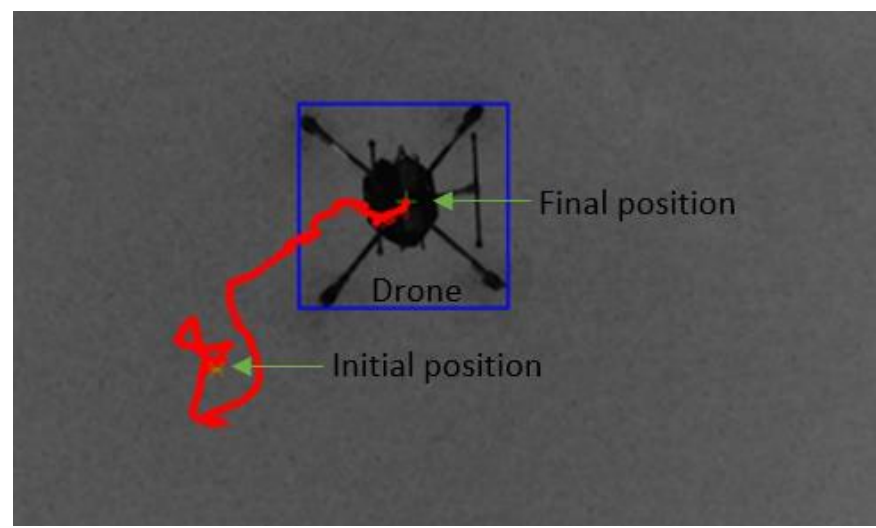

Fig. 6. Sample frame capture of object detection algorithm in MATLAB

From the trajectory data, the square of the error (SSE) was calculated using Eq. 1 for all three cases, for the entire 30second video (i.e., total of 1800 frames). The results are tabulated in Table 1 .

As previously stated, the SSE calculation provides a mean of comparing the three cases in terms of the degree in which the UAV deviated from its original location. A lower SSE means a more stable UAV during station-keeping.

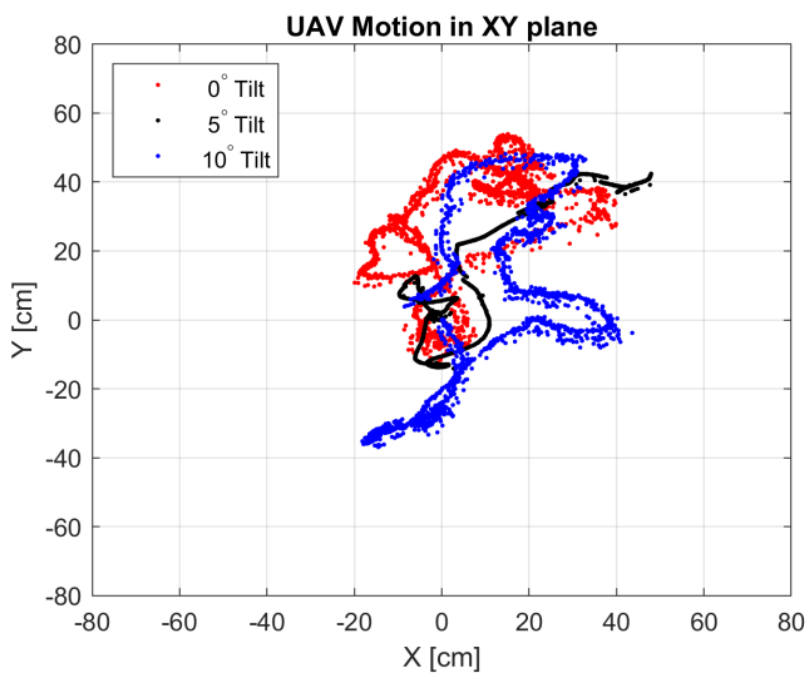

Fig. 7. Comparison of the drone trajectory for all three cases $(0,5,10 \mathrm{deg}$.)

As evidenced by the SSE results, the UAV becomes more stable during station-keeping with an increased propeller tilt angle. It is also worth noting that the change in SSE between $0^{\circ}$ and $5^{\circ}$ is much larger when compared to that between $5^{\circ}$ 
and $10^{\circ}$, which may indicate a diminishing effect on stability at higher tilt angles.

TABLE I

CALCULATED SUM OF THE SQUARE OF THE ERROR (SSE)
\begin{tabular}{|c|c|}
\hline Tilt Angle [Deg] & SSE $\left[\mathbf{c m}^{2}\right]$ \\
\hline $0^{\circ}$ & 1280.80 \\
\hline $5^{\circ}$ & 984.54 \\
\hline $10^{\circ}$ & 979.14 \\
\hline
\end{tabular}

\section{CONCLUSION}

The results obtained demonstrate that tilting the propellers of a UAV inward improved the flight stability during stationkeeping by a factor of approximately 1.3 when compared to no tilt angle, as evidenced by the overall decrease in SSE. Note that the improvement in flight stability from $5^{\circ}$ to $10^{\circ}$ was relatively small when compared to the stability improvement from $0^{\circ}$ to $5^{\circ}$, which represents a diminishing effect that may indicate there may be an optimal propeller tilt angle that maximizes the stability of the UAV during station-keeping. However, further testing at more propeller tilt angles is necessary to verify this postulation. Thus, the development of an experimental setup in which the propeller tilt angle can be actively modified is the focus of future work.

\section{ACKNOWLEDGMENT}

The authors gratefully acknowledge the support of the Department of Energy (DoE) sponsored Consortium for Integrating Energy Systems in Engineering and Science Education (CIESESE), the José Domingo Pérez School of Engineering at Universidad Ana G. Méndez - Gurabo, and the Puerto Rico Energy Center (PREC).

\section{REFERENCES}

[1] V. Jorge, R. Granada, R. Maidana, et al. "A Survey on Unmanned Surface Vehicles for Disaster Robotics: Main Challenges and Directions." Sensors, Basel, Switzerland, vol. 19, 3702, Feb. 8, 2019.

[2] M. R. Haque, M. Muhammad, D. Swarnaker and M. Arifuzzaman, "Autonomous quadcopter for product home delivery," 2014 International Conference on Electrical Engineering and Information \& Communication Technology, Dhaka, 2014, pp. 1-5.

[3] S. Gudmundsson, General Aviation Aircraft Design: Applied Methods and Procedures, Elsevier Science, 2013.

[4] M. Hedayatpour, "Modeling and Control of Multi-Rotor UAVS: Design for Safety and Performance," M.S. thesis, Ind. Sys. Eng., University of Regina, Regina, Saskatchewan, 2019.

[5] A. C. Kahvecioglu and N. Alemdaroglu, "Optimal design of a miniature quad tilt rotor UAV," 2015 International Conference on Unmanned Aircraft Systems (ICUAS), Denver, CO, 2015, pp. 1118-1127.

[6] F. Kendoul, I. Fantoni and R. Lozano, "Modeling and Control of a Small Autonomous Aircraft Having Two Tilting Rotors," in IEEE Transactions on Robotics, vol. 22, no. 6, pp. 1297-1302, Dec. 2006.

[7] M. Ryll, H. H. Bülthoff and P. R. Giordano, "First flight tests for a quadrotor UAV with tilting propellers," 2013 IEEE International Conference on Robotics and Automation, Karlsruhe, 2013, pp. 295-302.

[8] H. Efraim, A. Shapiro, and G. Weiss, "Quadrotor with Dihedral Angle: On the Effects of Tilting the Rotors Inward," Journal of Intelligent Robot Systems, Vol. 80, pp. 313-324, 2015.
[9] H. Bezerra Diogenes and D. A. Dos Santos, "Modelling, Design, and Simulation of a Quadrotor with Tilting Rotors Actuated by a Memory Shape Wire," National Congress of Mechanical Engineering, CONEM 2016, Brazil, 2016.

[10]C. McClanahan, R. C. Bolam, Y. Vagapov and A. Anuchin, "Analysis of the Effects on the Pitching, Rolling and Yawing Rate of a V-tail Configured Quadcopter," 26th International Workshop on Electric Drives: Improvement in Efficiency of Electric Drives (IWED), Moscow, Russia, 2019.

[11]ArduPilot Dev Team: Mission Planner, http://ardupilot.org/planner/ index.html, 2019. 